\title{
Proportional-integral genetic algorithm controller for stability of TCP network
}

\author{
Mohammed Qasim Sulttan, Manal Hadi Jaber, Salam Waley Shneen \\ Energy and Renewable Energies Technology Center, University of Technology, Iraq
}

\begin{tabular}{|c|c|}
\hline Article Info & ABSTRACT \\
\hline $\begin{array}{l}\text { Keywords: } \\
\text { Active queue management } \\
\text { Genetic algorithm } \\
\text { Proportional-integral controller } \\
\text { TCP/IP }\end{array}$ & $\begin{array}{l}\text { The life development and increase the number of internet users imposed an } \\
\text { increase in data circulating on the internet network and then make } \\
\text { the network more congestion. As a result of all this, some problems arose such } \\
\text { as time delay in packets delivery, loss of packets, and exceed the buffer } \\
\text { capacity for the middle routers. To overcome those problems, transmission } \\
\text { control protocol and active queue management (TCP/AQM) have been used. } \\
\text { AQM is the main approach used to control congestion and overcome those } \\
\text { problems to improve network performance. This work proposes to use } \\
\text { the proportional-integral (PI) controller with a genetic algorithm (GA) as an } \\
\text { active queue manager for routers of the Internet. The simulation results show } \\
\text { a good performance for managing the congestion with using proportional- } \\
\text { integral genetic algorithm (GA-PI) controller better than the PI controller. } \\
\text { Copyright } \text { (c) } 2020 \text { Institute of Advanced Engineering and Science. } \\
\text { All rights reserved. }\end{array}$ \\
\hline \multicolumn{2}{|l|}{ Corresponding Author: } \\
\hline \multicolumn{2}{|c|}{$\begin{array}{l}\text { Salam Waley Shneen, } \\
\text { Energy and Renewable Energies Technology Center, } \\
\text { University of Technology, } \\
52 \text { Industry street, Baghdad, Iraq. } \\
\text { Email: salam_waley73@yahoo.com }\end{array}$} \\
\hline
\end{tabular}

\section{INTRODUCTION}

The safe and fast data transmission in communication systems without problems such as lengthy tardinesses in packets conveyance, loss of packets, increase the number of Internet users, and data congestion during the transmission process [1-5]. All these problems made researchers use modern methods and techniques to control and reduce these problems. The transmission control protocol (TCP) [6-9] is the first and major protocol of the Internet protocols group. It emerged in the premier network application in which it supplemented the internet protocol (IP), to become TCP/IP [10-12]. TCP equips authoritative, ordered, and check of error delivery of a flux of bytes between applications running on hosts communicating via an IP network. TCP is the more important protocol used to to internet traffic management; the TCP transmitters minify their rate of transmitting if there is a packet wastage detection [13-15]. The active queue management (AQM) [16-20] is an efficient technique to reveals introductory congestion and gives an early warning about the current internet network status by marking incoming packets before fulling the router queues, such technique is performed in the router to eschew crowding by creating packets projecting feedbacks through which crowding is notified to the source to take necessary action. The main objectives of using AQM are to marking the rate of inbound the traffic of a network, administer the queue length and with a low packet loss rate through the network resources [21-23].

Many of the techniques have been proposed in previous years illustrate a work of AQM [24-26], the oldest one is random early detection (RED) [27] which has been used to reducing the congestion during dropping the random packets. It turns out that the algorithm of RED is unsettled and sensitive to the effected factors of the network [28]. The RED algorithm depends on the fluid-based model to detect the congestion early time such as nonlinear fluid-flow model, to design a controller efficient, stabilized, and robust, the TCP and RED techniques requires to comprehend the system's dynamics [29-30]. 
Proportional-integral (PI) is one a control strategy uses to process plant system by using special techniques with a base-line like Ziegler-Nichols method for the tuning of the controllers. To create a system with good performance, the Ziegler-Nichols method is used to determine the controller parameters such as the proportional gain and integral gain constants [31-34]. The genetic algorithm (GA) is deemed as one of the useful controller techniques utilizing the basics of naturalist genetic systems to search for a universal solution of an improvement problem. The requisite idea from GA is to preserve a population of conceivable solution that develops and evolves with time through rivalry process and controlled variance. The GA has performed well in various fields such as modelling, power goodness evaluation, resource allocation and, adaptive tabling system, etc [35-37].

In [38] the researchers used the particle swarm optimization (PSO) as an optimization unit with proportional-integral-differential (PID) controller, in a large-delay network environment to improve TCP/AQM performance. In another work [39], the researchers are designed a robust controller (fractional PID) to control the congestion of TCP/AQM networks in a time-varying parameter, the proposed design has given good management to congestion of networks such as a stable queue length and less packet drop probability. SK Bisoy and PK Pattnaik in [40] proposed a new AQM by a novel-PD-type feedback controller to regulate the queue length with tiny oscillation. In [41] the authors proposed FPI controller with GA as tuning of those controller parameters to manage the congestion of TCP networks.

In this work, we proposed to use a PI controller as AQM of TCP network with GA to get an optimal PI controller gain to reduce the packet loss and queue length to achieve better performance for AQM scheme in TCP networks. The rest of this paper is organized as follows. Section 2 exhibit the system description that presents two subsections one of them is TCP/AQM networks and other is the GA and PI controller. Section 3 presents the simulation results. The conclusions were introduced in section 4 . The last section is section 5 include the references.

\section{SYSTEM DESCRIPTION}

This section contains the system parts and how each part work such as TCP network, PI controller and GA as an optimization tool.

\subsection{TCP/AQM network}

Many pieces of research illustrated the concept of TCP networks, a detailed of our work model was presented in this section depend on [42], the TCP network is based on a nonlinear fluid-flow dynamic model. The model is expressed with two non-linear differential (1) and (2), they show the work dynamic of TCP precisely with the rate of TCP window size and the rate of queue length.

$$
\begin{aligned}
& " \dot{w}(t)=\frac{1}{\frac{q(t)}{C}+T_{p}}-\frac{w(t)}{2} \frac{w(t-R(t))}{\frac{q(t-R(t))}{C}+T_{p}} p(t-R(t)) " \\
& " \dot{q}(t)=\left\{\begin{array}{lc}
-C+\frac{N(t)}{\frac{q(t)}{C}+T_{p}} w(t) & \text { if } q(t)>0 \\
\max \left\{0,-C+\frac{N(t)}{\frac{q(t)}{C}+T_{p}} w(t)\right\} & \text { if } q(t)=0
\end{array}\right.
\end{aligned}
$$

Where $w$ is the rate of TCP window size (measured in packets); $R$ is the transmission of full-trip time (measured in seconds) and equal to $\frac{q}{C}+T_{p} ; \mathrm{q}$ is the rate of queue length (measured in packets); $C$ is the capacity of the link (measured in packets/seconds); $N$ is the No, TCP links (load factor); $T_{p}$ is the promulgation delay (measured in seconds): $p$ is the packet sign probability, all these factors are assumed to be non-negative and it is the control input to reduce the transmitting rate and preserve the bottleneck queue extension. In (1), the two terms $1 / R$ (additive increase) and $w / 2$ (multiplicative decrease) in the congestion control algorithm are used to evaluate the rate of window size during the TCP flow, while (2) is the dynamics of the queue length accumulative as the rate of transmission overrides the capacity of the link.

The value of packet sign probability is between the lower boundary 0 and upper boundary 1 , the nonlinear time-delayed scheme and a saturated input are derived from (1) as in (3):

$$
\dot{w}(t)=\frac{1}{\frac{q(t)}{C}+T_{p}}-\frac{w(t)}{2} \frac{w(t-R(t))}{\frac{q(t-R(t))}{C}+T_{p}} \operatorname{sat}(p(t-R(t)))
$$


The saturated input is expressed by the nonlinearity equation as in (4):

$$
\operatorname{sat}(p(t-R(t)))=\left\{\begin{array}{cc}
1, & p(t-R(t)) \geq 1 \\
p(t-R(t)), & 0 \leq p(t-R(t))<1 \\
0, & p(t-R(t))<0
\end{array}\right.
$$

\subsection{The GA and PI controller (PIC)}

To fulfil the desired queue length and supply solid performance with delay effects and a saturated input, this work proposed to use a GA with PIC. A PIC generates the term $u(t)$ as a control input in (3) to warranty the stabilization of the system in (3). Moreover, the output error signal is expressed as $e(t)=q(t)-$ $q_{w} \mathrm{e}(\mathrm{t})=\mathrm{q}(\mathrm{t})-\mathrm{qw}$, where $q_{w}$ appears as a wished queue length. A PIC with an input $e(t)$ and an output $p(t-R(t))$ is defined as in (5):

$$
p(t-R(t))=K_{P}\left[e(t)+\frac{1}{T} \int_{0}^{t} e(\tau) d \tau\right]
$$

where: is the integrated time constant and $K_{p}$ : is proportional gain. In (5) can be expressed as:

$$
p(t-R(t))=K_{P} e(t)+K_{I} \int_{0}^{t} e(\tau) d \tau
$$

where $K_{I}$ represent the integral gain and equal to $K_{I}=\frac{K_{P}}{T}$

To know the measurement of the closed-loop control system and its performance, an integral and absolute value of error $\left(e_{i a}\right)$ is utilized as a thematic function as in (6).

$$
e_{i a}=\int_{0}^{\infty}|e(\tau)| d \tau
$$

As a certain fact, the function $e_{i a}$ will rely on the controller gains $K_{P}$ and $K_{I}$. A better PIC design requires a smaller value of $e_{i a}$. In a practical field, the design of the PIC is a process that encourages researchers to research and it relies on their experiments. The input output transfer function of the PIC is given by (8).

$$
\frac{122.8 s^{3}+3299 s^{2}+2455 s+3.252 e^{04}}{s^{4}+1.136 s^{3}+20.14 s^{2}+11.26 s+99.8}
$$

Various techniques used to optimize the performance of PIC such as fuzzy logic, particle swarm optimizer, grey wolf optimizer and genetic algorithm (GA). The GA will be exploited to obtain an optimal gain of PIC. GA is a synthetic optimization technique developed in the field of normal evolution executing an exploration of the search field. It is considered as an effective scheme for exploring or finding the global or near-global solution for complex improvement issues. In our work, the proposed GA search method used to find the optimal solutions for the PIC gains $\left(K_{P}\right.$ and $\left.K_{I}\right)$ for the AQM to backing the TCP such that the amount of $e_{i a}$ in (7) is less. Figure 1 offers the schematic shape for tuning of PIC parameters using GA.

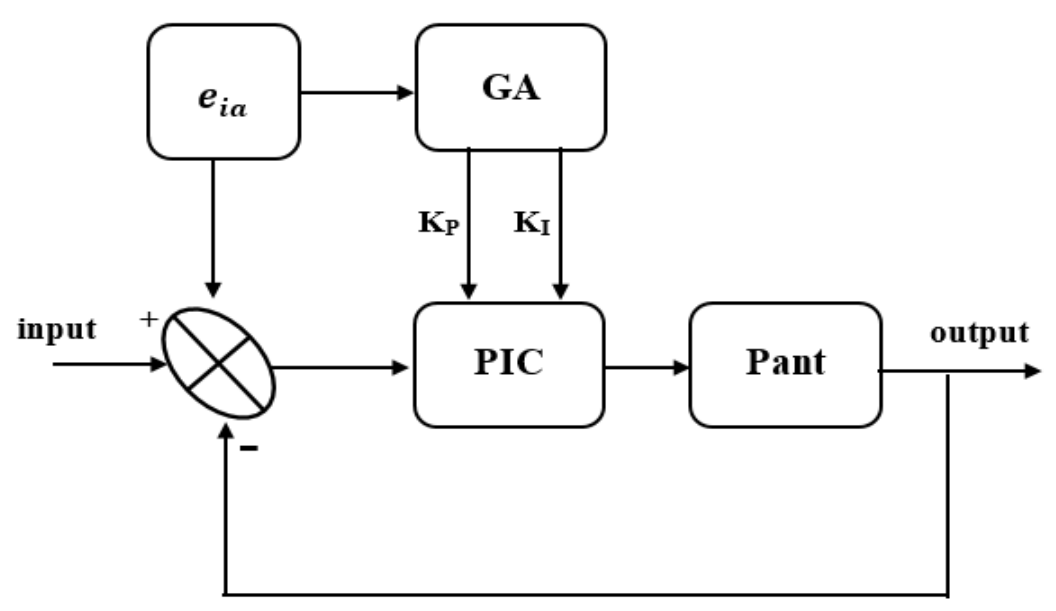

Figure 1. The schematic shape of PIC tuning using GA 
In a practical field, there is an important requirement, that the controller unit must be work as fast as possible, this can be achieved when the GA design with a small size of the incipient population. The incipient population is determined by encoding the PI factors, $K_{P}$ and $K_{I}$ through a binary concatenation define as a chromosome. The length of a concatenation relies on the desired accuracy which is about 4 mattresses. The desired bits series is calculated by (9):

$$
2^{n-1}<\left(U_{b}-L_{b}\right) \times 10^{4} \leq 2^{n}-1
$$

where $n$ is the number of bits, $U_{b}$ and $L_{b}$ are the upper bounds and lower bounds of PI factors respectively.

Each chromosome is subject to converting process to evaluate it, each chromosome is converted from binary strings to real values (PI factors values) and substitute into a $e_{i a}$. The converting process of each chromosome is done by (10):

$$
C_{v}=L_{b}+\operatorname{Dec}(\text { subseries }) \times \frac{\left(U_{b}-L_{b}\right)}{2^{n}-1}
$$

where $C_{v}$ is the real numbers of PI factors, Dec(subseries) represents the decimal values of bit series that calculate in (9).

Whole fitness values and their congruous chromosome are going through three processes (selection, crossover and mutation). In the selection process, the higher the value of fitness, the greater the opportunity of choosing a member of the population. The crossover process comes after the selection process by choosing a single-point crossover. The two mating chromosomes are randomly chosen one cut-point and interchange the right part of the two parents to create progeny. The third operation is a mutation, the mutation prohibits the algorithm from falling in a local minimum and preserving variety in the population. ordinarily, a minimal mutation rate must be selected because the maximal mutation rate may cause the searching process will be random process.

After finishing the three processes, the next generation starting by converting (decode) the binary concatenations in every chromosome in the population into real values. A new group of PI factors is sent to the PIC system to calculate the new value of fitness. This procedure will pass through the three processes sequentially, this repeated to reach a better value of fitness and this achieved at the close of generations. Figure 2 shows the flowchart of a GA [43].

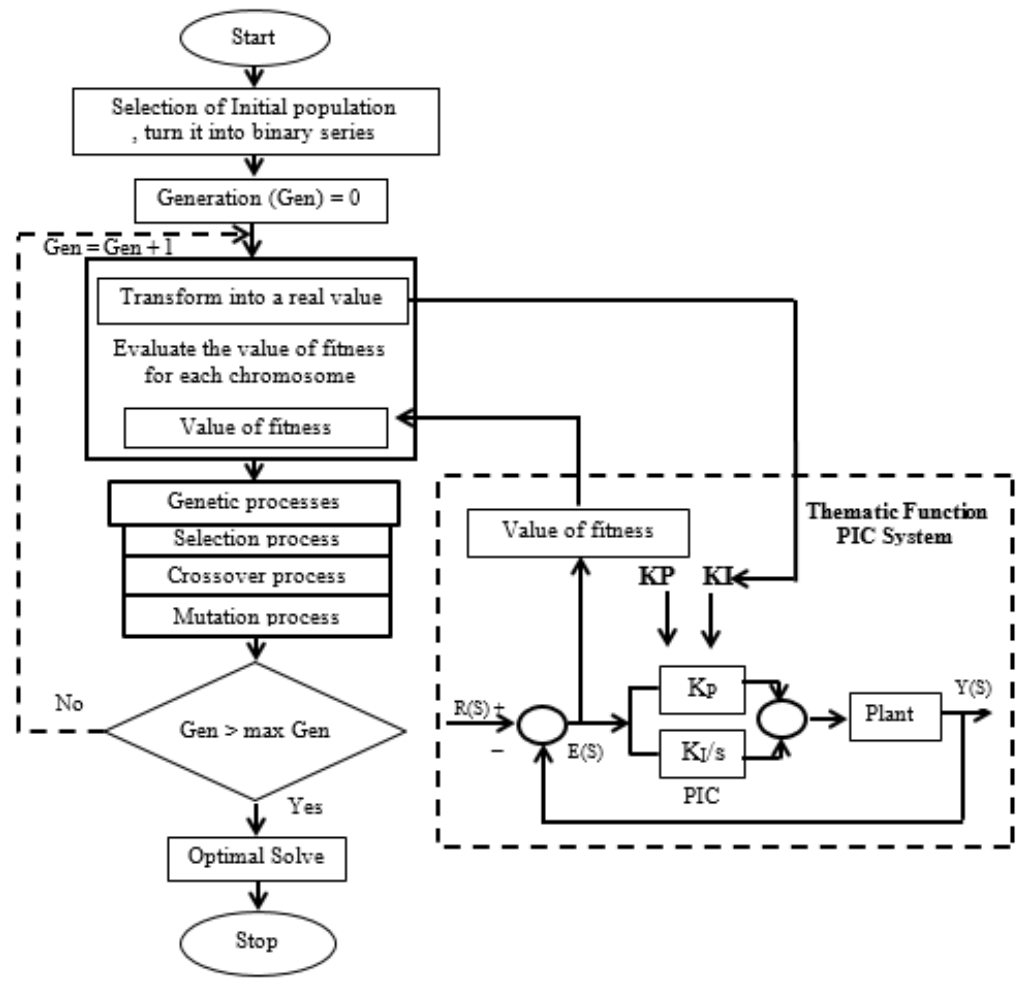

Figure 2. The flowchart of a GA

Int J Elec \& Comp Eng, Vol. 10, No. 6, December 2020 : 6225 - 6232 


\section{SIMULATION RESULTS}

In this section, we explain the effectiveness of the assumed GA-PIC for TCP network by compared and evaluated it with the PIC technique to managing the congestion to improve the efficiency of TCP/AQM system. A simulation program (MATLAB) was used to determine the competence of the supposed controller and inspect its susceptibility to averting network crowding. Now suppose we are work in a network consist of a single bottleneck (15 Mb/s in $25 \mathrm{msec}$ ) connects two routers connected to a group of homogenous TCP transmitters and receivers. According to Eqs. 1 and 2, we suppose the factors of the network as follow: the full-trip time $=0.25 \mathrm{sec}$, the capacity of the link $=15 \mathrm{Mb} / \mathrm{sec}$, load factor $=60$, promulgation delay $=0.2 \mathrm{sec}$, the desired queue size $=300$ packets and maximum queue length in the router of sending $=700$ packets.

Figure 3 exhibit the behavior of the system without a controller on the AQM. It is noted that the queue is very crowded and cannot follow the requested queue length. Figure 4 shows the Simulink model for TCP/AQM with PIC, Figure 5 presents a comparison between the performance of TCP/AQM network with PIC and with GA-PI, the network with GA-PI appeared good results than the network with PIC only such as lower rise time, overshoot and settling time as in Table 1.

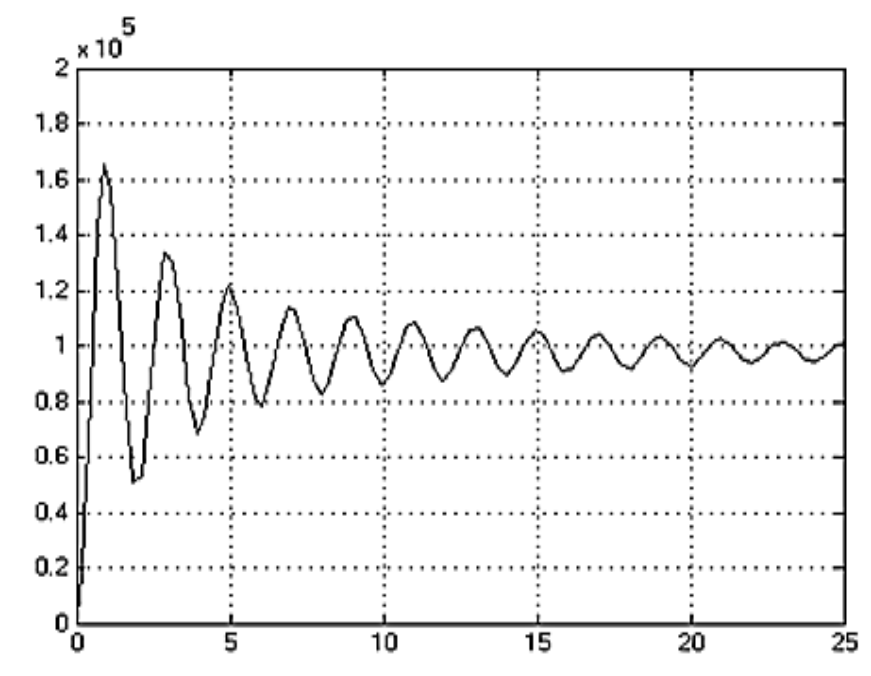

Time offset: 0

Figure 3. The response of the system without a controller

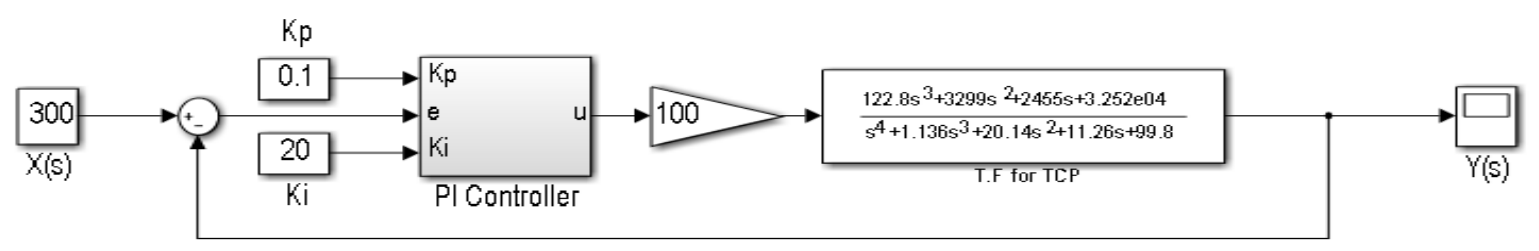

Figure 4. The Simulink model for TCP/AQM with PIC

Table 1. The results for the comparison between AQM schemes

\begin{tabular}{cccc}
\hline Settling Time $(\mathrm{sec})$ & Over Shoot $(\%)$ & Rise Time $(\mathrm{sec})$ & AQM scheme \\
\hline $\mathbf{0 . 0 3 2 2}$ & 3.49 & 0.0032 & GA_PI Controller \\
$\mathbf{0 . 0 7 2 7}$ & 8.24 & 0.0079 & PI Controller \\
\hline
\end{tabular}

Figure 6 shows the convergence of fitness values vs. iterations (generations), the values of the best fitness and mean fitness is $6.82421 e^{-5}, 7.2117 e^{-5}$ respectively. Figure 7 shows the parameters of GA-PIC that use to optimizing the performance TCP/AQM network, the best performance is achieved in iteration number 51with the controller gains $K_{P}=10.987$ and $K_{I}=6.145$, the thematic function (objective function) $e_{i a}=1.171 e^{-4}$. 


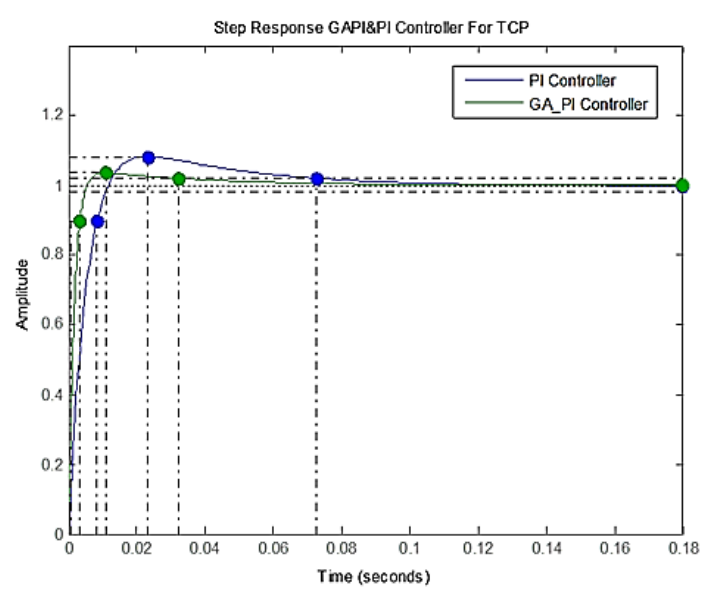

Figure 5. System response with PIC and GA-PI controller

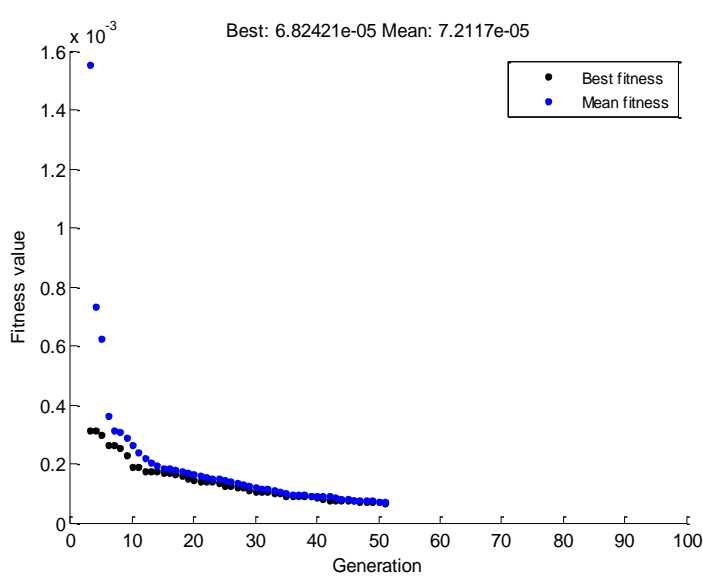

Figure 6. The convergence of fitness values vs. Iterations

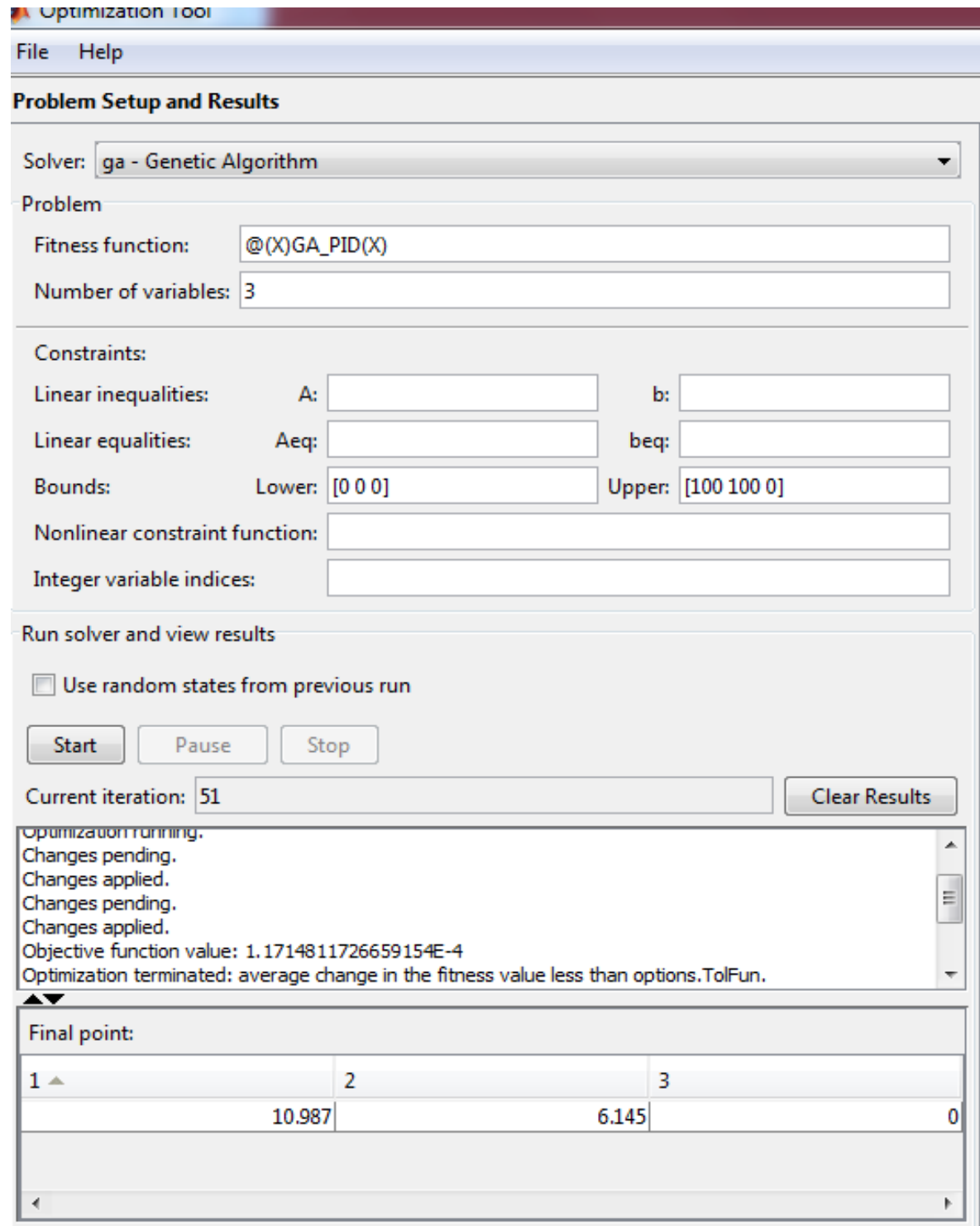

Figure 7. The parameters of GA-PIC 


\section{CONCLUSOIN}

In this paper, GA-PI controller as AQM scheme for TCP network was proposed to avert network congestion. The proposed algorithm achieved good results comparing with PIC such as faster rise time and settling time also a lower percentage of overshoot. The simulation results manifested that the proposed algorithm to manage the congestion is superior to the traditional AQM scheme that depends on PIC.

\section{REFERENCES}

[1] Afanasyev, Alexander, et al. "Host-to-host congestion control for TCP," IEEE Communications surveys and tutorials, vol. 12, no. 3, pp. 304-342, 2010.

[2] Sulttan, M. Q., "Impact of using infinity-norm with initial radius on performance and complexity of SD algorithm in MIMO systems," International Journal Of Computers and Technology, vol. 15, no. 6, pp. 6857-6864, 2016.

[3] M, Rendy, et al. "Performance analysis of tunnel broker through open virtual private network," TELKOMNIKA Telecommunication, Computing, Electronics and Control, vol. 17, no. 3, pp. 1185-1192, 2019.

[4] Rizzo, Luigi., "PGMCC: A TCP-friendly single-rate multicast congestion control scheme," ACM SIGCOMM Computer Communication Review, vol. 30, no. 4, pp. 17-28, 2000.

[5] Sulttan, M. Q., "Enhancement of K-Best Sphere Detection Algorithm Performance in MIMO Systems," IOP Conference Series: Materials Science and Engineering, vol. 518, no. 5, 2019.

[6] Okokpujie, K., et al., "Comparative analysis of the performance of various active queue management techniques to varying wireless network conditions," International Journal of Electrical and Computer Engineering (IJECE), vol. 9, no. 1, pp. 359-368, 2019.

[7] Nigar, N., and Azim, M. A., "Fairness Comparison of TCP Variants over Proactive and Reactive Routing Protocol in MANET," International Journal of Electrical and Computer Engineering (IJECE), vol. 8, no. 4, pp. 2199-2206, 2018.

[8] Wheeb, A. H., "Performance evaluation of UDP, DCCP, SCTP and TFRC for different traffic flow in wired networks," International Journal of Electrical and Computer Engineering (IJECE), vol. 7, no. 6, pp. 3552-3557, 2017.

[9] Hertiana, S. N., Kurniawan, A., and Pasaribu, U. S., "Effective Router Assisted Congestion Control for SDN," International Journal of Electrical and Computer Engineering (IJECE), vol. 8, no. 6, pp. 4467-4476, 2018.

[10] Sarolahti, Pasi, M. Kojo, and K. Raatikainen, "F-RTO: an enhanced recovery algorithm for TCP retransmission timeouts," ACM SIGCOMM Computer Communication Review, vol. 33, no. 2, pp. 51-63, 2003.

[11] Cai, Lin, et al., "Performance analysis of TCP-friendly AIMD algorithms for multimedia applications," IEEE Transactions on Multimedia, vol. 7, no. 2, pp. 339-355, 2005.

[12] Kure, O., "Basic Internet Protocol (IP)," TELEKTRONIKK, vol. 93, pp. 3-11, 1997.

[13] Samsuddin, MI Bin, et al., "The evaluation of AdBlock technique implementation for enterprise network environment," International Journal of Electrical and Computer Engineering (IJECE), vol. 13, no. 3, pp. 1102-1109, 2019.

[14] K. Wijaya, M. H H. Ichsan, and S. R. Akbar, "UDP pervasive protocol implementation for smart home environment on MyRIO using LabVIEW," International Journal of Electrical and Computer Engineering (IJECE), vol. 8, no. 1, pp. 113-123, 2018.

[15] Dostálek, Libor, and Alena Kabelová, "Understanding TCP/IP," Packt, Birmingham, 2006.

[16] Okokpujie, K. O., et al., "Novel Active Queue Management Scheme for Routers in Wireless Networks," International Journal on Communications Antenna and Propagation, vol. 8, no. 1, pp. 53-61, 2018.

[17] Alaoui, S. B., Tissir, E. H., and Chaibi, N., "Active queue management based feedback control for TCP with successive delays in single and multiple bottleneck topology," Computer Communications, vol. 117, pp. 58-70, 2018.

[18] Rezaee, A. A., and Pasandideh, F., "A fuzzy congestion control protocol based on active queue management in wireless sensor networks with medical applications," Wireless Personal Communications, vol. 98, no. 1, pp. 815-842, 2018.

[19] Kua, J., Nguyen, S. H., Armitage, G., and Branch, P., "Using active queue management to assist IoT application flows in home broadband networks," IEEE Internet of Things Journal, vol. 4, no. 5, pp. 1399-1407, 2017.

[20] Li, Z. H., Liu, Y., and Jing, Y. W., "Active Queue Management Algorithm for TCP Networks with Integral Backstepping and Minimax," International Journal of Control, Automation and Systems, vol. 17, no. 4, pp. 1059-1066, 2019.

[21] Sauter, Martin, "Communication systems for the mobile information society," John Wiley \& Sons, 2006.

[22] Eryilmaz, Atilla, and R. Srikant, "Fair resource allocation in wireless networks using queue-length-based scheduling and congestion control," IEEE/ACM transactions on networking, vol. 15, no. 6, pp. 1333-1344, 2007.

[23] G. Yuan, G. He, and J. C. Hou, "On exploiting traffic predictability in active queue management," Proceedings. Twenty-First Annual Joint Conference of the IEEE Computer and Communications Societies, vol. 3, 2002.

[24] Rahme, Sandy, Yann Labit, and Frédéric Gouaisbaut, "Sliding mode observer for anomaly detection in TCP/AQM networks," 2009 Second International Conference on Communication Theory, Reliability, and Quality of Service, IEEE, 2009.

[25] Gong, Yixi, et al., "Fighting the bufferbloat: On the coexistence of AQM and low priority congestion control," Computer Networks, vol. 65, pp. 255-267, 2014.

[26] Samsuddin, MI Bin, et al., "The evaluation of AdBlock technique implementation for enterprise network environment," Indonesian Journal of Electrical Engineering and Computer Science (IJEECS), vol. 13, no. 3, pp. 1102-1109, 2019.

[27] Patel, Sanjeev, P. Gupta, and G. Singh. "Performance measure of Drop tail and RED algorithm," 2nd International Conference on Electronic Computer Technology, 2010. 
[28] Sagfors, Mats, et al., "Queue management for TCP traffic over 3G links," IEEE Wireless Communications and Networking, vol. 3, 2003.

[29] Weigle, Michele C., Kevin Jeffay, and F. Donelson Smith, "Delay-based early congestion detection and adaptation in TCP: impact on web performance," Computer Communications, vol. 28, no. 8, pp. 837-850, 2015.

[30] O, Kennedy, et al., "Comparative analysis of the performance of various active queue management techniques to varying wireless network conditions," International Journal of Electrical and Computer Engineering (IJECE), vol. 9, no. 1, pp. 359-368, 2018.

[31] Shneen, S. Waley, C. Mao, and D. Wang, "Advanced optimal PSO, Fuzzy and PI controller with PMSM and WTGS at $5 \mathrm{~Hz}$ side of generation and $50 \mathrm{~Hz}$ Side of Grid," International Journal of Power Electronics and Drive Systems (IJPEDS), vol. 7, no. 1, pp. 173-129, 2016.

[32] S. W. Shneen, H. Sh. Dakheel, Z. B. Abdulla, "Design and implementation of variable and constant load for induction motor," International Journal of Power Electronics and Drive System (IJPEDS), vol. 11, no. 2, pp. 762-773, 2020.

[33] S. S. Sabah, and N. M. Kaittan, "Grey wolf optimizer based fuzzy-PI active queue management design for network congestion avoidance," Indonesian Journal of Electrical Engineering and Computer Science (IJEECS), vol. 18, no. 1, pp. 199-208, 2020.

[34] S. S. Waley, "BBO tuned PI control for three phases rectifier," J. Sci. Eng. Res, vol. 5, pp. 471-479, 2018.

[35] Attiya, Adnan Jabbar, Y. Wenyu, and Salam Waley Shneen, "PSO_PI Controller of Robotic Grinding Force Servo System," Indonesian Journal of Electrical Engineering and Computer Science (IJEECS), vol. 15, no. 3, pp. 515-525, 2015.

[36] Waley, Salam, Chengxiong Mao, and Nasseer K. Bachache, "Biogeography based optimization for tuning FLC controller of PMSM," International Conference in Swarm Intelligence, 2015.

[37] A. A. Jabbar, Y. Wenyu, and S. S. Waley, "Compared with PI, Fuzzy_PI \& PSO_PI Controllers of Robotic Grinding Force Servo System," TELKOMNIKA Indonesian Journal of Electrical Engineering, vol. 16, no. 1, pp. 65-74, 2015.

[38] Wang, X., Wang, Y., Zhou, H., and Huai, X., "PSO-PID: a novel controller for AQM routers," IFIP International Conference on Wireless and Optical Communications Networks, 2006.

[39] Hamidian, H., and Beheshti, M. T., "A robust fractional-order PID controller design based on active queue management for TCP network," Indonesian Journal of Electrical Engineering and Computer Science (IJEECS), vol. 49, no 1, pp. 211-216, 2018.

[40] Bisoy, S. K., and Pattnaik, P. K., "Design of feedback controller for TCP/AQM networks," International Journal Engineering Science and Technology, vol. 20, no. 1, pp, 116-132, 2017.

[41] Di Fatta, et al., "A genetic algorithm for the design of a fuzzy controller for active queue management," IEEE Transactions on Systems, Man, and Cybernetics, Part C (Applications and Reviews), vol. 33, no. 3, pp. 313-324, 2003.

[42] Hollot, C. V., Misra, V., Towsley, D., and Gong, W., "Analysis and design of controllers for AQM routers supporting TCP flows," IEEE Transactions on automatic control, vol. 47, no. 6, pp. 945-959, 2002.

[43] S. S. Waley, M. Q. Sulttan, and M. H. Jaber, "Variable speed control for 2Ph-HSM in RGS: a comparative simulation study," International Journal of Electrical and Computer Engineering (IJECE), vol. 10, no. 3, pp. 2285-2295, 2020 